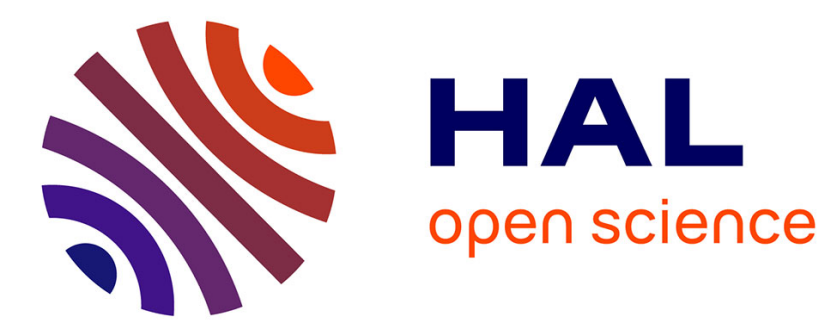

\title{
Gel-like elasticity in glass-forming side-chain liquid-crystal polymers
}

O. Pozo, D. Collin, H. Finkelmann, D. Rogez, P. Martinoty

\section{To cite this version:}

O. Pozo, D. Collin, H. Finkelmann, D. Rogez, P. Martinoty. Gel-like elasticity in glass-forming sidechain liquid-crystal polymers. Physical Review E: Statistical, Nonlinear, and Soft Matter Physics, 2009, 80 (3), 10.1103/PhysRevE.80.031801 . hal-00431643

\section{HAL Id: hal-00431643 \\ https://hal.science/hal-00431643}

Submitted on 22 Apr 2020

HAL is a multi-disciplinary open access archive for the deposit and dissemination of scientific research documents, whether they are published or not. The documents may come from teaching and research institutions in France or abroad, or from public or private research centers.
L'archive ouverte pluridisciplinaire HAL, est destinée au dépôt et à la diffusion de documents scientifiques de niveau recherche, publiés ou non, émanant des établissements d'enseignement et de recherche français ou étrangers, des laboratoires publics ou privés. 


\title{
Gel-like elasticity in glass-forming side-chain liquid-crystal polymers
}

\author{
O. Pozo, ${ }^{1, *}$ D. Collin, ${ }^{1}$ H. Finkelmann, ${ }^{2}$ D. Rogez, ${ }^{1}$ and P. Martinoty ${ }^{1}$ \\ ${ }^{1}$ Institut Charles Sadron, UPR 22, 23 rue du Loess, 67034 Strasbourg Cedex 2, France \\ ${ }^{2}$ Institut für Makromoleculare Chemie, Stefan-Meier-Str. 31, 79104 Freiburg, Germany
}

(Received 27 February 2009; published 22 September 2009)

\begin{abstract}
We study the complex shear modulus $G$ of two side-chain liquid-crystal polymers (SCLCPs), a methoxyphenylbenzoate substituted polyacrylate (thereafter called $\mathrm{PAOCH}_{3}$ ), and a cyanobiphenyl substituted polyacrylate supplied by Merck (thereafter called LCP105) using a piezoelectric rheometer. Two methods of filling the cell are used: (a) a capillary method, which can be used only at high temperature because of the low value of the viscosity, and (b) the classical one, thereafter called compression method, which consists in placing the sample between the two slides of the cell and to bring them closer. By filling the cell at high temperature either with the compression or the capillary method, we show that the response of both compounds is liquidlike $\left(G^{\prime} \sim f^{2}\right.$ and $G^{\prime \prime} \sim f$, where $f$ is the frequency) for temperatures higher than a certain temperature $T_{0}$ and gel-like $\left(G^{\prime} \sim\right.$ const, $\left.G^{\prime \prime} \sim f\right)$ below $T_{0}$. This change in behavior from the conventional flow response to a gel-like response, when approaching the glass transition, is observed for nonsliding conditions and for very weak-imposed shear strains. It can be explained by a percolation-type mechanism of preglassy elastic clusters, which correspond to long-range and long-lived density fluctuations that are frozen at the time scale of the experiment. The sample response is therefore the sum of two contributions: one is due to the flow response of the polymer melt and the other to the elastic response of the network formed by the preglassy elastic clusters. By filling the cell below $T_{0}$ with the compression method, both compounds exhibit a gel-type behavior by gently bringing closer the slides of the cell and an anomalous low-frequency behavior characterized by $G^{\prime}$ $=$ const and $G^{\prime \prime}=$ const by increasing the pressure used to bring closer the slides of the cell. A compressionassisted aggregation of the preglassy elastic clusters can explain both the increase in the low-frequency elastic plateau when the sample thickness is decreased and the anomalous low-frequency behavior. Further evidence for the existence of these elastic clusters is provided by the following results: (a) the nonlinear response of the samples as a function of the strain amplitude, which can be explained by the Payne effect, and (b) the aggregation effects, which can be mimicked by a polydimethylsiloxane melt filled with silica particles, the silica particles playing the role of the preglassy elastic clusters. All these observations show that $\mathrm{PAOCH}_{3}$ is not a macroscopically solidlike material with an unconventional type of elasticity, as claimed by Mendil et al. [Phys. Rev. Lett. 96, 077801 (2006)]. The gel-type behavior observed here on two SCLCPs $\left(\mathrm{PAOCH}_{3}\right.$ and LCP105) and previously on some conventional flexible polymers (atactic polystyrene, poly- $n$-butylacrylate) seems to be a generic effect of the glass transition. The presence of the preglassy elastic clusters questions the widely accepted hypothesis of ergodicity in the supercooled state.
\end{abstract}

DOI: 10.1103/PhysRevE.80.031801

PACS number(s): 64.70.pj, 61.30.Vx, 83.85.Cg

\section{INTRODUCTION}

Many liquids, whether they are simple liquids or polymeric liquids, do not crystallize when they are cooled but become very viscous, turning into solid glasses when their glass transition temperature $T_{G}$ is reached. Understanding the glass transition is of interest to several fields from soft matter to material sciences and biophysics. However, despite more than half a century of intense research, the glass transition remains mysterious and is still one of the great enigmas of contemporary physics [1].

It has long been recognized that glass formation is associated with heterogeneities (long-lived spatially extended clusters) which cause deviations from pure liquid behavior [2-4]. Understanding the nature, length scale, and time scale of these heterogeneities is one of the most fundamentally

\footnotetext{
*Present address: IPC-Institute for Polymers and Composites, University of Minho, Campus de Azurem, Guimaraes, Portugal 4800-058.
}

interesting issues in the field of the glass transition when it is approached from above.

Heterogeneity has been observed in light-scattering experiments by Fischer's group [5-11] and by the Nevada group [12] on polymer melts as well as on low molecular weight glass-forming liquids. Probably the most prominent feature revealed by dynamic light scattering is the existence of long-range density fluctuations with correlation lengths up to $\sim 2000 \AA$ and correlation times which can be $10^{7}$ times longer than the $\alpha$-relaxation time of glass-forming liquids and polymer melts. These long-range density fluctuations, which, close to $T_{G}$, do not relax within the time scale of the experiment, make the system apparently nonergodic. X-ray scattering [8], polarized hole-burning [4,13], single-molecule spectroscopy $[14,15]$, NMR $[16,17]$, and dielectric relaxation spectroscopy $[18,19]$ also provide further evidence for heterogeneity.

The first rheological experiments showing deviations from a liquid behavior to an elastic behavior for the real part $G^{\prime}$ of the shear modulus have been reported by us, first on side-chain liquid-crystal polymers (SCLCPs) $[20,21]$ and 
then on conventional flexible polymers [22]. Initially, the observations made on the SCLCPs were assumed to be a liquid-crystalline effect, an assumption which has been dropped in view of the experimental results obtained later on conventional flexible polymers [22,23].

The experiments we performed later by decreasing the temperature after filling the cell by capillarity at high temperature have shown that this deviation is characterized by a change in $G^{\prime}$ from the $f^{2}$ behavior observed at high temperature (typically $\sim 100{ }^{\circ} \mathrm{C}$ above the glass transition temperature) to a low-frequency elastic behavior occurring below a certain temperature $T_{0}[22,23]$. This unexpected change in behavior, from liquid to gel, has only been observed for sufficiently small sample thicknesses (typically a few tens of microns). It has been explained in terms of a "percolation" of preglassy elastic clusters, which correspond to long-range density fluctuations associated with the glass transition and which are long-lived and therefore frozen at the frequencies of measurement $[22,23]$. The measurements were taken with the shear piezorheometer we developed over more than a decade and applied to the study of the dynamic properties of polymers [20-23], liquid crystal elastomers [24-31], uniaxial magnetic gels [32], suspensions of magnetic particles [33], and polyelectrolyte films [34]. These experiments were performed as a function of sample thickness with strong anchoring conditions on the sample-bearing glass slides and with very small applied strains in order to prevent slipping processes. A macroscopic description of the preglassy elastic clusters observed by light scattering as well as by piezorheometry is given in [35].

Recently, Noirez [36] and Mendil et al. [37-39] also reported deviations from liquid behavior using a conventional rheometer. In particular, they have performed dynamical shear measurements in the isotropic and nematic phases of a methoxy-phenylbenzoate SCLCP, called $\mathrm{PAOCH}_{3}$, showing that the terminal regime is characterized by a frequencyindependent behavior of both $G^{\prime}$ and $G^{\prime \prime}$, and have concluded that the compound is a macroscopically solidlike material with a novel elasticity [39].

This statement has been challenged by Collin and Martinoty [40], who presented an alternative explanation suggesting that the solidlike behavior observed by Mendil et al. could be due to the compression of the sample stemming from the cell filling method they used. This method (thereafter called compression method) consists in placing the sample between the two plates of the cell and to bring them closer, thereby provoking an unavoidable compression of the sample. However, Mendil et al. argued that the compound we have studied - a cyanobiphenyl SCLCP called LCP105was chemically different from their own $\left(\mathrm{PAOCH}_{3}\right)$ and also that the capillary method we used to fill the cell is probably not a stress-free method [41].

The aim of this paper is to clarify the debate and to answer the basic question to know whether a SCLCP is a solid with an unconventional type of elasticity or a liquid in which an elastic network associated with preglassy elastic clusters gradually emerges when approaching the glass transition. For this purpose, we have synthesized a $\mathrm{PAOCH}_{3}$ polymer melt, similar to the one studied by Mendil et al., and performed our shear experiments by using a compression method to fill the cell, analogous to that of Mendil et al. By using this method, we have checked the influence on the mechanical properties, of the filling temperature, of the strength of the pressure applied on the sample when one fills the cell, and of the surface treatment of the sample-bearing silica slides. We have also checked the evolution of the sample response as a function of time. Finally, additional experiments were also performed by filling the cell with the capillary method, as we had done previously in $[22,23]$.

To provide further evidence of the preglassy elastic clusters, we have expanded this study in two directions: (a) in the nonlinear regime, where the results can be interpreted in terms of the Payne effect, and (b) in the linear regime, to probe on a polymer melt filled with solid particles, whether the preglassy elastic clusters can be mimicked by solid particles.

More specifically, when the cell is filled at high temperature by gently bringing closer the sample-cover silica slide to the sample-bearing silica slide, we show that the sample response is liquidlike for temperatures higher than some temperature $T_{0}$ and that the $f^{2}$ response of $G^{\prime}$ is replaced when the temperature is reduced by a low-frequency elastic response occurring below $T_{0}$. This change in behavior shows that the compound is not solidlike with an unconventional elasticity characterized by $G^{\prime}=$ const and $G^{\prime \prime}=$ const in contrast to the statement of Mendil et al. Moreover, the additional experiments we have performed by filling the cell at high temperature by using the capillary method lead to the same type of behavior, which demonstrates the validity of the capillary method to obtain significant results, in contrast to the claim by Mendil et al. [41].

We also show that the pressure applied on the sample by bringing closer the sample-cover silica slide to the samplebearing silica slide plays a crucial role in the mechanical response of the sample. In particular, below $T_{0}$, a gel-like behavior is observed by gently bringing closer the samplecover silica slide to the sample-bearing silica slide and a solidlike behavior by increasing the pressure used to bring closer the silica slides. We show that this solidlike behavior is very robust as a function of time and can persist several weeks above $T_{0}$. We have also taken measurements on LCP105, the compound studied in $[23,40]$, which according to Mendil et al. [41] should exhibit a mechanical response different from that of $\mathrm{PAOCH}_{3}$. The results obtained for LCP105 confirm those found for $\mathrm{PAOCH}_{3}$, indicating that the choice of the compound is not crucial, in contrast to the statement of Mendil et al. [41,42].

A compression-assisted aggregation of the preglassy elastic clusters present in the melt can explain the increase in the low-frequency elastic plateau when the sample thickness is decreased, and the anomalous solidlike behavior which has been observed by Mendil et al. [39].

We have also studied the influence of the applied shear strain on the mechanical response of the samples, and found that this response can be explained by the Payne effect, which provides an additional argument in favor of the existence of the preglassy elastic clusters.

Finally, we show that the aggregation effects can be mimicked with a conventional polydimethylsiloxane (PDMS) melt filled with silica particles, the silica particles playing the role of the preglassy elastic clusters. 
(a)<smiles>COc1ccc(OC(=O)c2ccc(OCCCCCCOC(=O)C(F)(F)F)cc2)cc1</smiles>

(b)

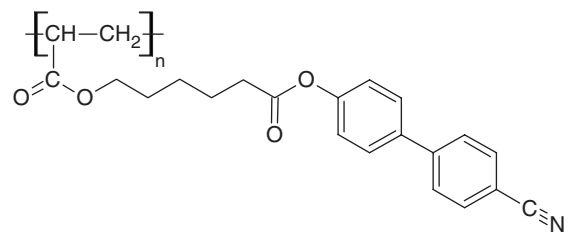

FIG. 1. Chemical structure of the side-chain liquid crystalline polymers studied: (a) $\mathrm{PAOCH}_{3}$ and (b) $\mathrm{LCP} 105$.

\section{MATERIALS AND METHODS}

\section{A. Compound}

The polymer we investigated has been synthesized in the Institut für Makromolekulare Chemie, Freiburg, Germany. It is a methoxy-phenylbenzoate substituted polyacrylate (thereafter called $\mathrm{PAOCH}_{3}$ ) similar to the one studied by Mendil et al. [39]. Its chemical composition is represented in Fig. 1(a). The compound had a molecular weight of $24000 \mathrm{~g} / \mathrm{mol}$ and a polydispersity index $I \sim 1.7$. It showed a nematic phase, a smectic- $A$ phase, and a glassy state. The phase transition temperatures determined by differential scanning calorimetry (DSC) were $T_{G} \sim 22{ }^{\circ} \mathrm{C}, T_{N-S m A} \sim 92{ }^{\circ} \mathrm{C}$, and $T_{N I} \sim 116{ }^{\circ} \mathrm{C}$. They were obtained from the position of the DSC peaks by extrapolating to cooling rate zero the data taken at cooling rates of $2,4,9$, and $16 \mathrm{~K} / \mathrm{min}$. The glass transition temperature was obtained as the temperature increased at $2 \mathrm{~K} / \mathrm{min}$.

We have also taken measurements on a cyanobiphenyl substituted polyacrylate supplied by Merck and referenced as LCP105. This compound already studied in $[23,40]$ had a molecular weight of $11680 \mathrm{~g} / \mathrm{mol}$ and a polydispersity index of 1.8. The phase transition temperatures were $T_{G}$ $=38{ }^{\circ} \mathrm{C}$ and $T_{N I}=120{ }^{\circ} \mathrm{C}$. Its chemical composition is given in Fig. 1(b).

\section{B. Technique}

The shear piezorheometer is a plate-plate rheometer that uses piezoelectric ceramics vibrating in the shear mode to apply a slight displacement on one side of the sample and to measure the transmitted stress on the other side of the sample. The strain imposed on the sample is given by

$$
\varepsilon=\frac{\delta}{L} \propto V_{\text {in }}=\left(V_{0}\right)_{\text {in }} e^{i \omega t},
$$

where $V_{i n}$ is the voltage applied to the emitting ceramic, $\delta$ is the shear displacement induced by the ceramic, and $L$ is the thickness of the sample. The stress measured on the receiving ceramic is given by

$$
\sigma=\frac{F}{S} \propto V_{\text {out }}=\left(V_{0}\right)_{\text {out }} e^{i(\omega t+\varphi)},
$$

where $F$ is the force transmitted by the sample of surface area $S$ and $V_{\text {out }}$ is the voltage given by the ceramic. The phase $\varphi$ can vary from zero (perfect elastic solid) to $\pi / 2$ (Newtonian liquid). The complex shear modulus of the sample is given by

$$
G=\frac{\sigma}{\varepsilon} \propto \frac{\left(V_{0}\right)_{\text {out }}}{\left(V_{0}\right)_{\text {in }}} e^{i \varphi} .
$$

The piezorheometer allows the complex shear modulus $G$ to be measured over sample thicknesses ranging from $\sim 10$ to $\sim 100 \mu \mathrm{m}$ for a liquid (up to $\sim 500 \mu \mathrm{m}$ for a soft solid), over a wide frequency range (from $10^{-2}$ to $10^{4} \mathrm{~Hz}$ in the most favorable case) and by applying very weak strains $\left(10^{-5}-10^{-3}\right)$. In practice, the sample is placed between two glass slides, one of which being stuck to the emitting ceramic and the other to the receiving ceramic. The faces of the glass slides on which the ceramics are glued are grounded so that there is no voltage applied to the sample. The high Curie temperature $\left(350^{\circ} \mathrm{C}\right)$ of the ceramics used (PZT PIC 255 from PI-POLYTEC) and the absence of temperature dependence of $d_{15}$, which is the only piezoelectric coefficient involved in the shear measurements, explain why the piezorheometer can work at temperatures up to $180{ }^{\circ} \mathrm{C}$ without loosing its sensitivity. It is therefore possible to perform reliable experiments in a wide range of temperature, in contrast to the remark made by Mendil et al. in [42].

In the present study, the strain $\varepsilon$ varied from a few $10^{-5}$ (linear-response regime) to a few $10^{-3}$ (non-linear-response regime), the temperature of the cell was regulated to within a few $10^{-2}{ }^{\circ} \mathrm{C}$, the parallelism of the silica slides was adjusted to within $\pm 10^{-4}$ rad and the cell was calibrated using liquids and soft solids (elastomers and gels) whose rheological properties are well known. The temperature dependence of the gap between the sample-bearing and sample-cover silica slides, which must be known for taking measurements as a function of temperature, is below $0.13 \mu \mathrm{m} / \mathrm{K}$. To avoid any slip of the sample on the sample-bearing and sample-cover silica slides, the latter were submitted to a UV irradiation in a flux of oxygen. The aim of this procedure was to remove the residual traces of organic molecules and to induce silanol groups on the silica surface of the slides, which can interact with the sample molecules. The influence of the anchoring conditions of the sample on the sample-bearing and samplecover silica slides surfaces will be discussed in detail in Secs. III A and III B.

The cell was loaded by placing the compound between the silica slides and then by bringing closer the sample-cover silica slide to the sample-bearing silica slide. For most of our experiments, the cell was filled at high temperature and the measurements taken by decreasing the temperature with a slow cooling rate of $\sim 1 \mathrm{~K} /$ hour. For the other experiments, the procedure used will be indicated in the text. All the experiments were performed with $\varepsilon \leq 10^{-4}$, except for the measurements taken as a function of $\varepsilon$. 


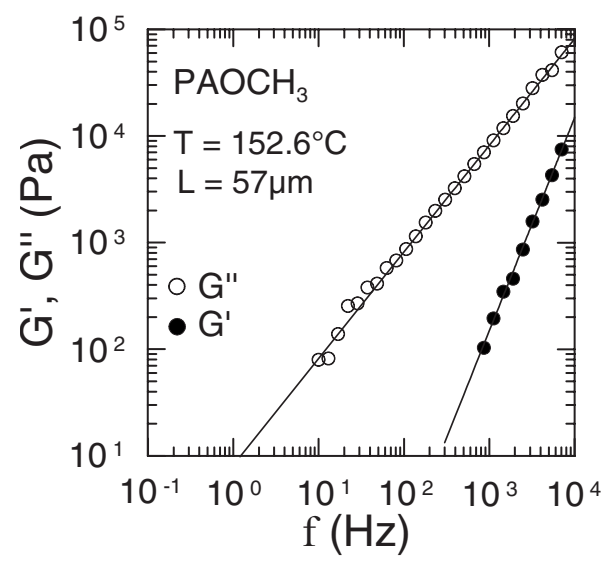

FIG. 2. Frequency dependence of the real $G^{\prime}$ and imaginary $G^{\prime \prime}$ part of the shear modulus of $\mathrm{PAOCH}_{3}$ showing that the SCLC melt exhibits at $T=152.6{ }^{\circ} \mathrm{C}$ the conventional flow behavior. The sample thickness $L$ was $57 \mu \mathrm{m}$. The straight lines represent the behaviors of $G^{\prime \prime}$ and $G^{\prime}$ proportional to $f$ and $f^{2}$, respectively.

\section{RESULTS AND DISCUSSION}

\section{A. Behavior of the complex shear modulus $G$ observed when the cell was loaded at high temperature using the compression method}

Before presenting our experimental results, we recall that the classical picture of a polymer in the molten state is that of a system which flows in the hydrodynamic regime (also called the terminal regime) [43]. This flow behavior is characterized by

$$
\begin{aligned}
G^{\prime} & =\omega^{2} \eta \tau, \\
G^{\prime \prime} & =\omega \eta,
\end{aligned}
$$

where $G^{\prime}$ and $G^{\prime \prime}$ represent the real and imaginary parts, respectively, of the complex shear modulus and $\omega=2 \pi f$. $\omega$ is the angular frequency, $f$ is the frequency, $\eta$ is the viscosity of the polymer, and $\tau$ is its terminal relaxation time.

\section{Behavior as a function of frequency and temperature for a given thickness}

Figure 2 shows the frequency variation in $G^{\prime}$ and $G^{\prime \prime}$ we have obtained for $\mathrm{PAOCH}_{3}$ after loading the gap at $152.6^{\circ} \mathrm{C}$ (i.e., at $\sim 130{ }^{\circ} \mathrm{C}$ from $T_{G}$ ) by gently bringing closer the sample-cover silica slide to the sample-bearing silica slide. It can be seen that $G^{\prime} \sim f^{2}$ and $G^{\prime \prime} \sim f$, which shows that the response of the compound is liquidlike. This result is in contrast with the solidlike behavior $\left(G^{\prime} \sim\right.$ const, $G^{\prime \prime} \sim$ const $)$ reported by Mendil et al. [39] for the same temperature range.

The experiments conducted by decreasing the temperature show that there is a temperature $T_{0}$ below which the $f^{2}$ response of $G^{\prime}$ is spontaneously replaced by a low-frequency elastic response (elastic plateau) that will be denoted thereafter by $G_{P L}$. It has been checked that $T_{0}$ is independent of the temperature at which the cell was filled, which shows that the elastic response does not stem from a compression of the sample due to the reduction in the gap between the sample-bearing and sample-cover silica slides, as the tem-

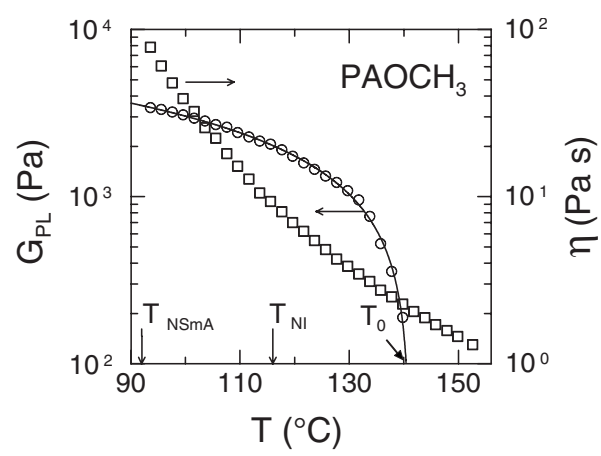

FIG. 3. Temperature-dependence of the elastic plateau $G_{P L}$ of $\mathrm{PAOCH}_{3}$ and of the viscosity $\eta$ deduced from the $G^{\prime \prime}$ behavior. The cell was filled at a temperature $\left(T=152.6^{\circ} \mathrm{C}\right)$ for which the sample showed the conventional flow behavior and the measurements taken by decreasing the temperature. $T_{N I}$ and $T_{N S m A}$ correspond to the nematic to isotropic and nematic to smectic- $A$ phase transition temperatures determined by DSC measurements. The variation in the sample thickness as a function of temperature has been taken into account to plot the curve. The thicknesses associated with the smaller and higher $G_{P L}$ values in the curve are $\sim 54$ and $\sim 48 \mu \mathrm{m}$, respectively. The solid line corresponds to a fit of the $G_{P L}$ data made with equation $G_{P L}=G_{0}\left(T_{0}-T\right)^{t}$, where $T_{0}$ is the temperature at which the elastic plateau appears. Unlike $G_{P L}$, no anomaly at $T_{0}$ can be observed in the $\eta$ behavior.

perature decreases. Figure 3 shows the temperature $T_{0}$ at which the elastic plateau appears and the variation in $G_{P L}$ as a function of temperature. It can be seen that $T_{0}$ is located above the nematic to isotropic phase transition $T_{N I}$, which indicates that the elastic plateau is not associated with this transition. Unlike $G^{\prime}$, there is no anomaly in the $G^{\prime \prime}$ behavior at $T_{0}$. This is illustrated by the same figure, which shows the temperature dependence of the viscosity deduced from the $G^{\prime \prime}$ measurements. A typical example of the behavior of $G^{\prime}$ and $G^{\prime \prime}$ as a function of frequency observed at any temperature located below $T_{0}$ is given in Fig. 4. It can be seen that the low-frequency part of the spectrum is characterized by

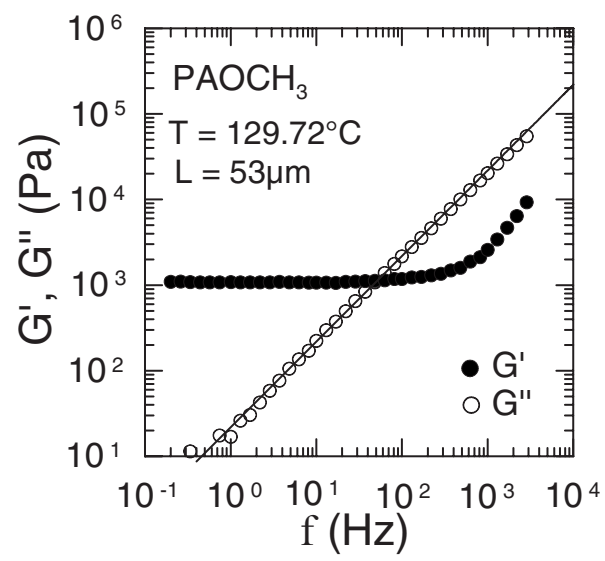

FIG. 4. Frequency dependence of the real $G^{\prime}$ and imaginary $G^{\prime \prime}$ part of the shear modulus of $\mathrm{PAOCH}_{3}$ at $T \sim 129{ }^{\circ} \mathrm{C}$ showing that the mechanical response of the sample is gel-like $\left(G^{\prime} \sim\right.$ const and $G^{\prime \prime} \sim f$ ) in the low-frequency range. The sample thickness $L$ was $53 \mu \mathrm{m}$. The straight line represents the behavior for $G^{\prime \prime}$ proportional to $f$. 
$G^{\prime} \sim$ const and $G^{\prime \prime} \sim f$ and not with the solidlike behavior $\left(G^{\prime} \sim\right.$ const, $G^{\prime \prime} \sim$ const $)$ reported by Mendil et al. in [39].

The low-frequency behavior $\left(G^{\prime}=\right.$ const, $\left.G^{\prime \prime} \sim f\right)$ we observe here in the molten state of $\mathrm{PAOCH}_{3}$ is similar to that we have previously observed in the molten state of LCP105 [23]. Therefore, the low-frequency behavior does not depend on the chemical composition of the compounds and seems to be universal for these structured materials, in contrast to the statement of Mendil et al. [41].

As in our previous works [22,23], we also filled the cell by capillary effect at high temperature. At high temperature, we observed a flow behavior similar to the one observed by filling the cell with the compression method, confirming that the response of the sample is liquidlike. Also and similarly to the compression method, the experiments performed by decreasing the temperature showed the occurrence of an elastic response for $G^{\prime}$, which occurs below a temperature $T_{0}$, and a conventional behavior for $G^{\prime \prime}$ in the whole temperature range investigated. In short, both methods used for filling the cell at high temperature lead to a similar behavior. This eliminates the doubts raised by Mendil et al. in [41] about the validity of the capillary method to obtain significant results.

\section{Analysis and discussion}

a. Preglassy elastic clusters. All our observations are similar to those we made previously on various polymer melts [22,23] and can therefore be interpreted along the same lines. As we have proposed in $[22,23]$, the elastic behavior observed in $G^{\prime}$ can be explained by the presence of longrange density fluctuations, which are associated with the glass transition and which are kinetically frozen in our frequency range because of their very slow relaxation times. These elastic clusters resemble the so-called Fischer's clusters observed by light scattering on several glass formers, including polymer melts [5]. When the temperature decreases, the number and the size of these clusters increase so that they can "percolate" in the shear cell, giving rise to a low-frequency elastic response $\left(G^{\prime} \sim\right.$ const, $\left.G^{\prime \prime} \sim 0\right)$, which is then added to the flow response of the polymer melt $\left(G^{\prime}\right.$ $\left.\sim f^{2}, G^{\prime \prime} \sim f\right)$. In other words, the observed response is the sum of two contributions: one (liquidlike) associated with the polymer and the other (solidlike) associated with the glass transition, leading to an apparent gel-type behavior $\left(G^{\prime} \sim\right.$ const, $\left.G^{\prime \prime} \sim f\right)$. This gel-like behavior does not indicate the existence of some generic elasticity of the polymer melt. It should be noted that the presence of the elastic clusters implies a nonhomogeneous distribution of the free volume. The free volume in question is not the free volume between the molecules but the free volume between the clusters. This concept of free volume has also been proposed by de Gennes to explain the mosaic structure of glasses [44].

It should be noted that the transition from amorphous to glassy state via a gel-like response has also been observed in the amorphous phase of colloidal systems when approaching the glass transition [45]. However, the observed response exhibits a more complicated behavior than the one we observe here for the polymer systems. This difference in behav-

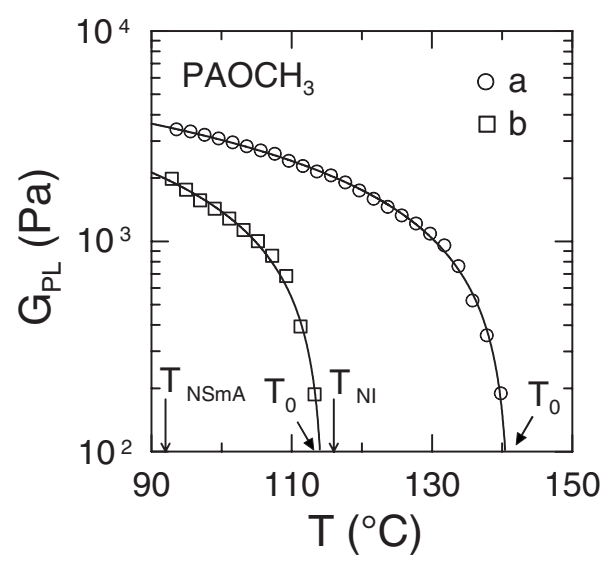

FIG. 5. Temperature dependence of the elastic plateau $G_{P L}$ for two $\mathrm{PAOCH}_{3}$ samples with different anchoring conditions. For each sample, $T_{0}$ is the temperature at which the elastic plateau appears. The solid lines are the results of a simultaneous fit of the data with equation $G_{P L}=G_{0}\left(T_{0}-T\right)^{t}$ using the same $t$ exponent. $t$ is $\sim 0.82$. For each sample, the thickness variation as a function of temperature has been taken into account to plot the curves. The thicknesses associated with the smaller and higher $G_{P L}$ values are $\sim 54$ and $\sim 48 \mu \mathrm{m}$, respectively (sample $a$ ), and $\sim 37$ and $\sim 34 \mu \mathrm{m}$, respectively (sample $b$ ).

ior comes from the fact that the relaxation frequency of the glassy mode is much lower for the polymer systems than for the colloidal glasses. As a result, the low-frequency glassy modes are completely decoupled from the polymer modes, whereas they are not decoupled from the colloidal modes. The question of modes addition has also been considered in block copolymers in the temperature range where microphase separation occurs. In that region, the microdomains may form a regular arrangement giving rise to a periodic structure, which disappears as the temperature is raised above a certain critical value, giving rise to an isotropic phase [46].

$b$. Influence of the anchoring conditions. The problem of the interaction between the sample and the sample-bearing silica slides is crucial since this interaction must be strong enough to avoid any slipping of the sample on both silica slides. The results presented above have been obtained by submitting the sample-bearing and sample-cover silica slides of the cell to a UV irradiation in a flux of oxygen. As already stressed in Sec. II, one aim of this process is to induce silanol groups on the silica surfaces of the slides, which can interact with the sample molecules, leading to a strong anchoring of the sample on the silica slides. Without this treatment, the melt can display either a decrease or the disappearance of the temperature $T_{0}$ at which the elastic plateau appears, depending whether the slipping of the sample on the silica slides is partial or total. The case of a partial slipping is illustrated by Fig. 5, which shows the $G_{P L}$ data (the squares) obtained by gently filling the cell at high temperature, after cleaning the sample-bearing and sample-cover silica slides of the cell without using the UV treatment. It can be seen that the reduction in the quality of the anchoring notably decreases the temperature $T_{0}$ at which the elastic plateau appears. It also 


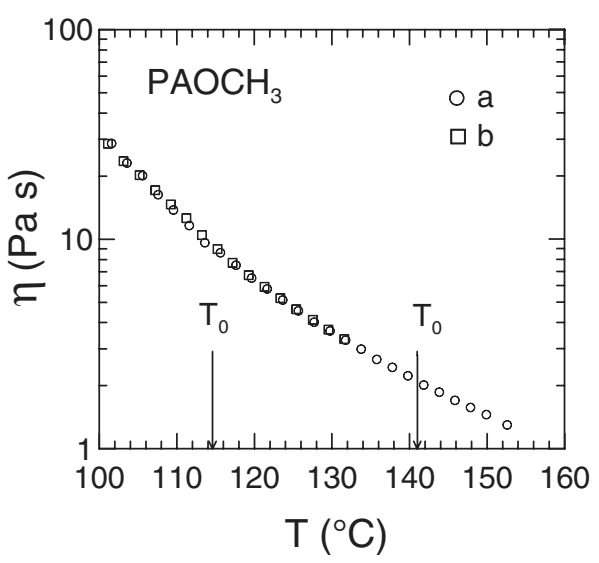

FIG. 6. Variation in the viscosity as a function of temperature for $\mathrm{PAOCH}_{3}$. The data come from the same experiments as those of Fig. 5 and are plotted using the same symbols. For each sample, the value of $T_{0}$ corresponds to the temperature at which the elastic plateau appears. No anomaly at $T_{0}$ can be observed in the $\eta$ behavior deduced from the $G^{\prime \prime}$ measurements. The thickness variation as a function of temperature has been taken into account to plot the curves. The thicknesses associated with the smaller and higher $\eta$ values are $\sim 57$ and $\sim 49 \mu \mathrm{m}$, respectively (sample $a$ ), and $\sim 39$ and $\sim 35 \mu \mathrm{m}$, respectively (sample $b$ ).

decreases the value of the elastic plateau but has no influence on the values of $G^{\prime \prime}$, as shown by the viscosity behavior reported on Fig. 6.

If we assume that the sample is composed of polymeric chains and of kinetically frozen density fluctuations (elastic clusters), the fact that $G^{\prime \prime}$ (and therefore the viscosity) remains unchanged with or without the UV surface treatment indicates that the polymeric chains do not slip. In contrast, the elastic clusters can slip more or less depending on the surface treatment. In our experiments, the polymeric chains are strongly anchored on the silica slides, whereas the elastic clusters are anchored on the polymer layer formed by the chains anchored on the silica slides. Our measurements show that the anchoring between the elastic clusters and the polymer chains is weaker than the anchoring between the polymer chains and the substrate.

The question is now to determine whether there are features that are independent of the anchoring conditions. The thermal dependence of the elastic plateau $G_{P L}$ obtained with the strong and the weak anchoring are reported on Fig. 5. Since both behaviors look like the formation of a physical gel, the data were analyzed with the equation $G_{P L}=G_{0}\left(T_{0}\right.$ $-T)^{t}$, where $T_{0}$ is the temperature for which the elastic plateau appears, $t$ is a critical exponent, and $G_{0}$ is a constant. The solid lines in Fig. 5 correspond to a simultaneous fit of the data, which means that exponent $t \sim 0.82$ is the same for both types of anchoring. This fit enables us to deduce the values of $G_{0}$ and $T_{0}$ for each anchoring quality and therefore to determine the values of $G_{P L}$ at the glass transition temperature $T_{G}$ and those of the difference $T_{0}-T_{G}$. To search for features that are independent of the anchoring conditions, we have plotted the data as a function of the reduced variables $G_{P L} / G_{P L}\left(T_{G}\right)$ and $\left(T_{0}-T\right) /\left(T_{0}-T_{G}\right)$. Figure 7 shows that this

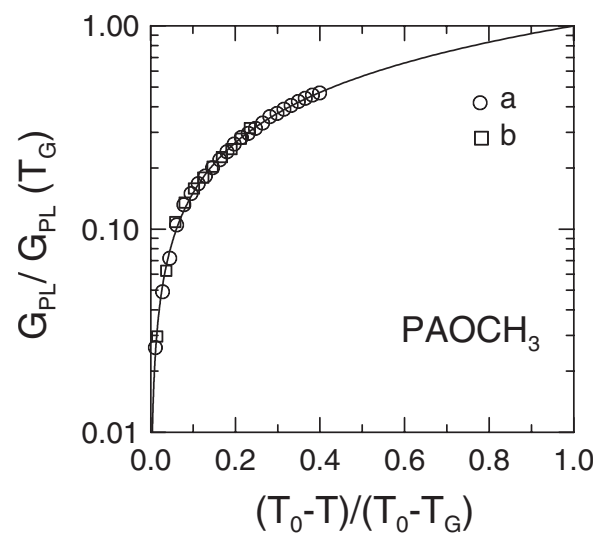

FIG. 7. $G_{P L}$ values normalized with respect to its value at $T_{G}$ as a function of the normalized temperature $\left(T_{0}-T\right) /\left(T_{0}-T_{G}\right)$. The reported data correspond to those of Fig. 5 using the same symbols. The solid line is the plot of equation $G_{P L} / G_{P L}\left(T_{G}\right)=\left[\left(T_{0}-T\right) /\left(T_{0}\right.\right.$ $\left.\left.-T_{G}\right)\right]^{t}$ with $t \sim 0.82$.

renormalization reveals a remarkable scaling with all the data falling onto a single plot, which indicates that the physics associated with the formation of the preglassy solidlike structure, is independent of the weak or strong anchoring conditions. This scaling behavior can be considered as an additional argument in favor of the presence of the preglassy elastic clusters.

It follows from the above discussion that a study of $G$ as a function of sample thickness is significant only if the anchoring conditions are the same for all the thicknesses. This study was not conducted in the present paper, but it was conducted in Refs. [22,23] associated with a conventional flexible polymer [22] and with LCP105 [23], respectively. In theses references, we have shown that the difference $T_{0}$ $-T_{G}$ increases when the thickness decreases, and that the increase in $T_{0}-T_{G}$ follows a power-law behavior for the thicknesses studied [22].

To sum up, our measurements taken after filling the cell at high temperature by gently bringing closer the sample-cover silica slide to the sample-bearing silica slide show that $\mathrm{PAOCH}_{3}$ is not a macroscopically solidlike material with a novel type of elasticity characterized by a frequencyindependent behavior of $G^{\prime}$ and $G^{\prime \prime}$ as claimed by Mendil et al. in [39]. At high temperature, the compound is liquidlike. At low temperature, below a certain temperature $T_{0}$, the compound becomes gel-like with a low-frequency elastic response $\left(G^{\prime}=\right.$ const $)$ associated with the glass transition, which is added to the flow response of the polymer melt.

In order to understand the anomalous solidlike behavior reported by Mendil et al., we have performed experiments as a function of the filling temperature and of the strength of the pressure used to bring closer the sample-cover silica slide to the sample-bearing silica slide. This is the subject of the next section. 


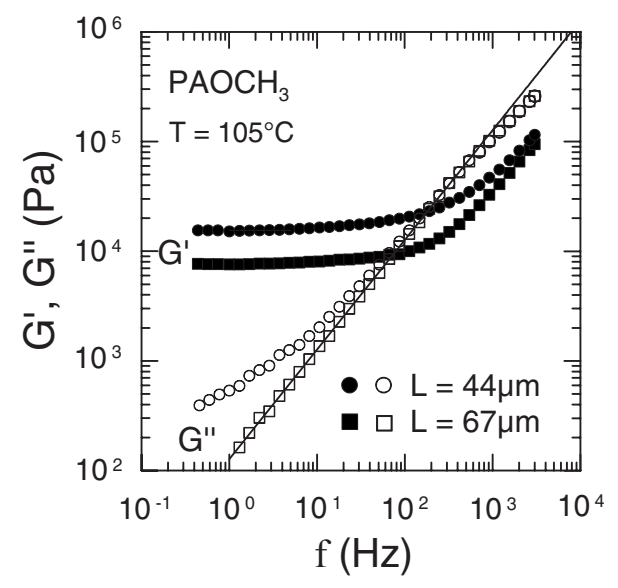

FIG. 8. Frequency dependence of the real $G^{\prime}$ and imaginary $G^{\prime \prime}$ part of the shear modulus of $\mathrm{PAOCH}_{3}$ at $T \sim 105{ }^{\circ} \mathrm{C}$. The cell was filled at this temperature. The reduction in the gap thickness produces a progressive shift from a gel-like behavior characterized by $G^{\prime} \sim$ const and $G^{\prime \prime} \sim f$ (as shown for $L=67 \mu \mathrm{m}$ ), to a solid-type behavior characterized by $G^{\prime} \sim G^{\prime \prime} \sim$ const (as shown for $L$ $=44 \mu \mathrm{m})$. The straight line represents the behavior for $G^{\prime \prime}$ proportional to $f$.

\section{B. Behavior of the complex shear modulus $G$ observed when the cell was loaded at low temperature with the compression method}

\section{Results}

As we will see now, the filling of the cell at low temperature (i.e., below $T_{0}$ ) with the compression method produces results, which (a) differ markedly from those observed after the cell was filled at high temperature (i.e., above $T_{0}$ ) and (b) depend dramatically on the strength of the pressure used to bring closer the sample-cover silica slide to the samplebearing silica slide.

By reducing the gap with the compression method, one observes first a gel-type apparent behavior $\left(G^{\prime} \sim\right.$ const, $G^{\prime \prime}$ $\sim f$ ), which is qualitatively similar to the one we have observed by decreasing the temperature after the cell was filled at high temperature, and then, for a stronger pressure applied on the sample, a solid-type behavior $\left(G^{\prime} \sim\right.$ const, $G^{\prime \prime}$ $\sim$ const) qualitatively similar to the one observed by Mendil et al. These observations clearly demonstrate that the mechanical response of the sample depends on the pressure applied on it by bringing closer the sample-cover silica slide to the sample-bearing silica slide. Typical examples of these behaviors are given by the data taken at $T=105^{\circ} \mathrm{C}$ and reported in Fig. 8.

Although the gel-type behavior obtained with the compression method is qualitatively similar to the one obtained without compressing the sample (i.e., by decreasing the temperature after the cell was filled at high temperature), the value of the elastic plateau at a given thickness is much higher for the compressed sample than for the one obtained without compressing the sample. In contrast, there is no change in the viscosity value. As an example, the $G^{\prime}$ value $\left(G^{\prime} \sim 7700 \mathrm{~Pa}\right)$ measured at $T=105{ }^{\circ} \mathrm{C}$ for $L=67 \mu \mathrm{m}$ (see Fig. 8) is higher than the value $\left(G^{\prime} \sim 2800 \mathrm{~Pa}\right)$ measured at

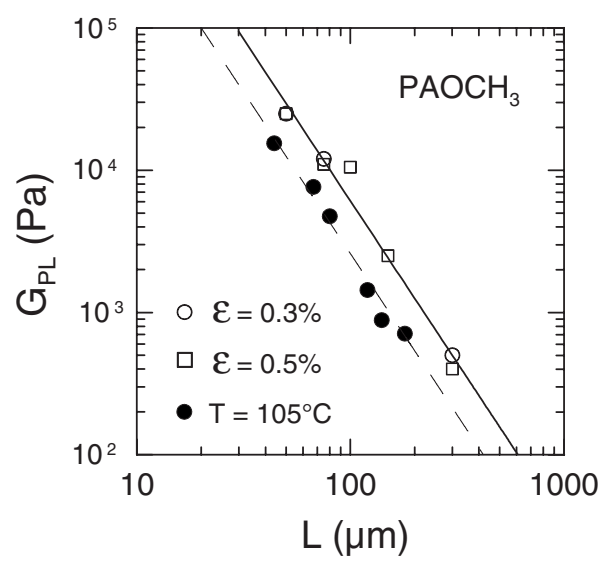

FIG. 9. Variation in the low-frequency elastic plateau of $\mathrm{PAOCH}_{3}$ as a function of sample thickness. The filled symbols correspond to our experiments, and the unfilled symbols correspond to the data obtained at $T=130{ }^{\circ} \mathrm{C}$ by Mendil et al. for different applied strains. The dashed and solid lines show that both our data and those of Mendil et al. can be described by a power law with a similar exponent.

the same temperature for $L=50 \mu \mathrm{m}$ (see Fig. 3), but the value of the viscosity remains the same $(\eta=18 \mathrm{~Pa} \mathrm{~s})$. This confirms that the behaviors of $G^{\prime}$ and $G^{\prime \prime}$ do not have the same physical origin, as suggested previously. $G^{\prime}$ is associated with the elastic response of the network formed by the preglassy elastic clusters and $G^{\prime \prime}$ with the flow response of the polymeric chains. The increase in $G^{\prime}$ indicates that the preglassy network is compressed by bringing closer the sample-cover silica slide to the sample-bearing silica slide even in the case where the pressure used to bring closer the slides is small.

Figure 9 shows the variation in the low-frequency elastic plateau as a function of thickness at $T=105^{\circ} \mathrm{C}$, which was observed by using the compression method. The filled and open symbols correspond to our measurements and to those of Mendil et al. [38], respectively. Only the measurements taken for the smallest values of the applied strains have been plotted in the case of the Mendil et al. experiments. Although the experimental conditions of the two experiments are not the same (the temperature, the anchoring conditions, the fact that the data of Mendil et al. are not in the linear-response regime, as we will see below), the thickness dependence of the elastic plateau, with a slope $\sim 2.2$, is nevertheless in qualitative agreement with that reported by Mendil et al. In contrast to the $G^{\prime}$ behavior, the $G^{\prime \prime}$ behavior we observe is however not consistent with the data reported by Mendil et al. Indeed, they observed a frequency-independent response for all the thicknesses studied, whereas in our case we observe this behavior for the smallest thickness studied (44 $\mu \mathrm{m}$ ) only and the classical $f$ behavior for the others thicknesses.

The last point concerns the anomalous solidlike behavior induced by the compression method as a function of time. The measurements, taken as a function of time at a given $T$, show that this anomalous behavior was very stable, with no significant variation over a three-month period. By increasing the temperature, the anomalous elastic behavior remains 
unchanged, as in the experiments of Mendil et al., and, when $T>T_{0}$, progressively disappears to recover the expected flow behavior.

\section{Analysis and discussion}

We have seen in Sec. III A that the elastic plateau observed on $G^{\prime}$ by decreasing the temperature after the cell was filled at high temperature by gently bringing closer the sample-cover silica slide to the sample-bearing silica slide or by capillarity can be explained by a percolation-type mechanism of the preglassy elastic clusters present in the melt, occurring at a temperature $T_{0}$. The sample response is therefore the sum of two contributions: one is due to the flow response of the polymer melt and the other to the elastic response of the network formed by the preglassy elastic clusters.

In the same way, the bringing closer the sample-cover silica slide to the sample-bearing silica slide at a temperature $T<T_{0}$, induces a reinforcement of the elastic network, as observed for example in Fig. 9, which can be explained by a compression-assisted aggregation of the preglassy elastic clusters. A weak compression of the sample leads to a gellike behavior $\left(G^{\prime} \sim\right.$ const, $\left.G^{\prime \prime} \sim f\right)$, and a strong compression leads to a solid-like behavior $\left(G^{\prime} \sim\right.$ const, $G^{\prime \prime} \sim$ const $)$. The number of clusters making the bridge between the two silica slides of the cell is therefore expected to be higher than the one associated with the temperature-assisted percolation. Their spatial distribution is also different since bringing closer the sample-cover silica slide to the sample-bearing silica slide induces a rearrangement of the preglassy clusters. The reinforcement of the network and the reorganization of the preglassy elastic clusters could explain why an elastic response can be observed above $T_{0}$, in the temperature range where the sample response is liquid type. This is illustrated by Fig. 1 of [40], which compares the data obtained at $T$ $=100.4{ }^{\circ} \mathrm{C}$ after the cell was filled at $T>T_{0}$ (liquidlike behavior), to those obtained after the cell was filled at $T<T_{0}$ (solidlike behavior) [47]. The sample studied was LCP105.

\section{Influence of the applied strain $\varepsilon$ on the mechanical response of a sample introduced into the cell at} low temperature (i.e., below $T_{0}$ ) with the compression method

We will now consider the influence of the applied strain $\varepsilon$ on the mechanical response of a sample introduced into the cell with the compression method. In this experiment the applied strain $\varepsilon$ was increased step by step and the sample response measured for each $\varepsilon$ value. The sample studied was LCP105, which exhibits a mechanical behavior similar to the one observed for $\mathrm{PAOCH}_{3}$, as shown previously. Figure 10 shows the data taken at $\varepsilon=8 \times 10^{-5}$ (original data), $8 \times 10^{-4}$, and $3 \times 10^{-3}$. This latter value corresponds to the smallest value used by Mendil et al. [38] in their experiments. The thickness of the sample was $30 \mu \mathrm{m}$, and the temperature was $84.2{ }^{\circ} \mathrm{C}$. The most striking observation is the existence of a low-frequency plateau in the $G^{\prime \prime}$ behavior, which is due to the compression method used to fill the cell, and which shows that the data are not in the linear-response regime even for the smallest strain used $\left(8 \times 10^{-5}\right)$. The progressive

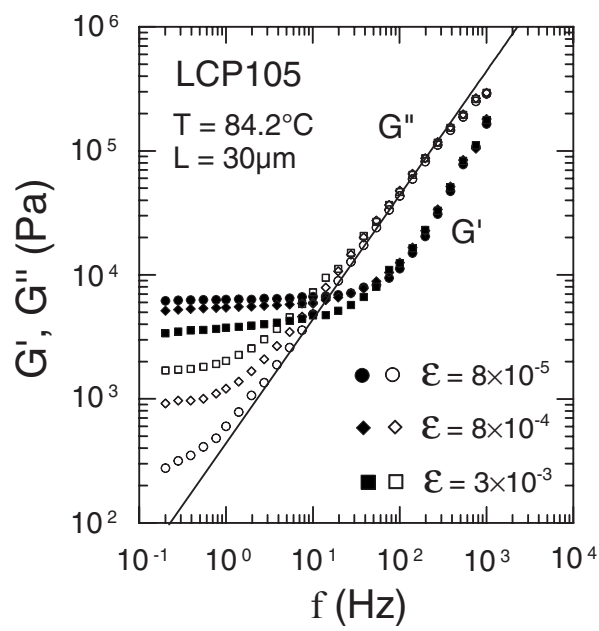

FIG. 10. Influence of the applied strain $\varepsilon$ on the mechanical response of LCP105 at $T \sim 84.2{ }^{\circ} \mathrm{C}$ showing, in the low-frequency range, that $G^{\prime}$ decreases and $G^{\prime \prime}$ increases when the applied strain increases. The sample thickness $L$ was $30 \mu \mathrm{m}$. The circles, the diamonds, and the squares correspond to $\varepsilon=8 \times 10^{-5}, \varepsilon=8 \times 10^{-4}$, and $\varepsilon=3 \times 10^{-3}$, respectively. The straight line represents the behavior for $G^{\prime \prime}$ proportional to $f$.

decrease in the value of the elastic plateau observed for $G^{\prime}$ when $\varepsilon$ increases leads to the same conclusion.

The decrease in $G^{\prime}$ and the simultaneous increase in $G^{\prime \prime}$ are reminiscent of the Payne effect when the strain is relatively low and corresponds to the beginning of the nonlinear-response regime. The simultaneous decrease in $G^{\prime}$ and $G^{\prime \prime}$ observed by Mendil et al. [38] could also be a feature of the Payne effect when the strain is very high even though these authors claim the contrary [42]. The Payne effect refers to the influence of the strain dependence on the dynamic properties of a polymer matrix filled with solid particles [48]. This effect has been observed in many systems, and two types of models have been proposed to explain it: one favors the filler-filler interactions leading to a filler network [49] and the other favors the filler-polymer matrix interactions [50]. Assuming that the Payne effect is associated with the breakdown of the filler network, the expressions for $G^{\prime}$ and $G^{\prime \prime}$ are then given by [49]

$$
\begin{gathered}
G^{\prime}(\varepsilon)=G_{\infty}^{\prime}+\frac{b}{1+u^{2}}, \\
G^{\prime \prime}(\varepsilon)=G_{\infty}^{\prime \prime}+c\left(\varepsilon_{c}\right)^{m} b \frac{u}{1+u^{2}},
\end{gathered}
$$

where $b, c$, and $m$ are constants. $b$ is given by $b=G_{0}^{\prime}-G_{\infty}^{\prime}$, where $G_{0}^{\prime}$ is the value of $G^{\prime}$ measured in the linear-response regime and $G_{\infty}^{\prime}$ is the value of $G^{\prime}$ when the strain $\varepsilon$ tends to infinity. $u$ is given by $u=\left(\varepsilon / \varepsilon_{c}\right)^{m}$, where $\varepsilon_{c}$ is the value of $\varepsilon$ when $u=1$.

The derivation of Eqs. (6) and (7) is based on the fact that the contacts between the fillers depend on the strain amplitude and break and reform at a particular rate. As a result, the number of contacts between the fillers decreases when the strain increases, leading to a decrease in $G^{\prime}$, which produces 


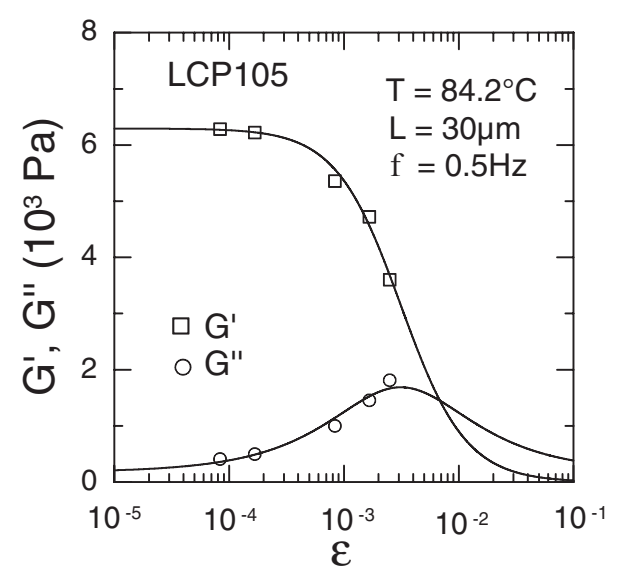

FIG. 11. Strain dependence of the real $G^{\prime}$ and imaginary $G^{\prime \prime}$ parts of the shear modulus of LCP105. The data come from the same experiments as those of Fig. 10. The solid lines are the results of a simultaneous fit of the $G^{\prime}$ and $G^{\prime \prime}$ data with Eqs. (6) and (7).

an energy dissipation giving rise to a peak in $G^{\prime \prime}$. It is assumed that the filler clusters are not appreciably stretched with increasing strain but rather break up almost immediately.

Figure 11 shows the variations of $G^{\prime}$ and $G^{\prime \prime}$ as a function of the strain amplitude for LCP105 at a frequency of $0.5 \mathrm{~Hz}$. It can be seen that our experimental results are faithfully represented by Eqs. (6) and (7). The solid lines correspond to a simultaneous fit of the data, and the resulting parameters are $G_{\infty}^{\prime \prime}=173 \mathrm{~Pa}, G_{0}^{\prime}=6295 \mathrm{~Pa}, c=40.7, \varepsilon_{c}=3.1 \times 10^{-3}$, and $m=0.76$. In this fit we have assumed that $G_{\infty}^{\prime} \sim 0 . G_{\infty}^{\prime}$ is the value obtained for the unloaded compound (in our case when the elastic preglassy clusters slip). From Fig. 14 of [23], it can be deduced that $G_{\infty}^{\prime} \sim 4 \times 10^{-2} \mathrm{~Pa}$ at a frequency of 0.5 $\mathrm{Hz}$, showing that it is correctly approximated by zero. Note that the value of $G_{\infty}^{\prime \prime}$ deduced from the fit $(173 \mathrm{~Pa})$ is consistent with the value $(\sim 200 \mathrm{~Pa})$ at $0.5 \mathrm{~Hz}$ deduced from Fig. 14 in [23]. We have also analyzed the $G^{\prime}$ and $G^{\prime \prime}$ data of Mendil et al. obtained in $\mathrm{PAOCH}_{3}$ for $300 \mu \mathrm{m}$, with the same Eqs. (6) and (7), in order to check whether the data can also be explained by the Payne effect. These data, taken at 1 $\mathrm{rad} / \mathrm{s}$, are those of Fig. 1 in [42] and are plotted in Fig. 12 as a function of the strain amplitude. The solid lines correspond to a simultaneous fit of the $G^{\prime}$ and $G^{\prime \prime}$ data, with the following resulting parameters, $G_{\infty}^{\prime \prime}=0, G_{0}^{\prime}=766 \mathrm{~Pa}, c=19.25, \varepsilon_{c}$ $=6 \times 10^{-3}$, and $m=0.80$. As for LCP105, we have assumed that $G_{\infty}^{\prime}=0$. The agreement between the data and the fit with Eqs. (6) and (7) can be considered to be reasonable, bearing in mind that this is a simultaneous fit of $G^{\prime}$ and $G^{\prime \prime}$ and that, for an equal experimental dispersion of the data, the relative accuracy of $G^{\prime \prime}$ is smaller than that of $G^{\prime}$ for the weakest strains. Note that the agreement between the data and the fit can be improved by using the plateau values of $G^{\prime}$ and $G^{\prime \prime}$ extracted from Figs. 1(a) and 1(b) of [38], as shown by the insert of Fig. 12.

Although the data for LCP105 are below the critical strain $\varepsilon_{c}$ and those for $\mathrm{PAOCH}_{3}$ essentially above it, it is possible to plot the $G^{\prime}$ data for both compounds on the same graph. This is illustrated by Fig. 13(a), which shows that all the data fall into a single curve given by

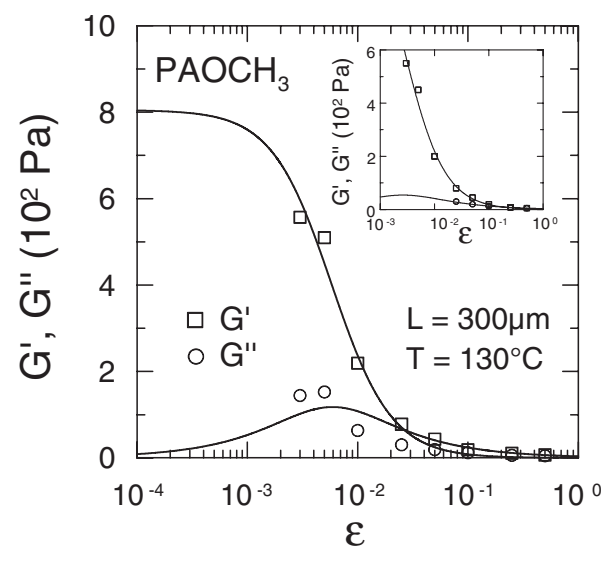

FIG. 12. Strain dependence of the real $G^{\prime}$ and imaginary $G^{\prime \prime}$ parts of the shear modulus of $\mathrm{PAOCH}_{3}$ at $T \sim 130{ }^{\circ} \mathrm{C}$. The sample thickness $L$ was $300 \mu \mathrm{m}$. The data are extracted from Fig. 1 of [42] (see text). The solid lines are the results of a simultaneous fit of the data with Eqs. (6) and (7). The inset shows the result of a similar fit performed on the data extracted from Figs. 1(a) and 1(b) of [38].

$$
\left(G^{\prime}-G_{\infty}^{\prime}\right) /\left(G_{0}^{\prime}-G_{\infty}^{\prime}\right)=1 /\left[1+\left(\varepsilon / \varepsilon_{c}\right)^{2 m}\right] .
$$

This master curve indicates that the effects observed in both compounds have the same physical origin, namely, the Payne effect, and this result provides an additional argument in favor of the existence of the preglassy elastic clusters in the melt. The quality of the master curve can be significantly improved by smoothing the original frequency-independent data reported in Fig. 1(a) of [38]. Indeed, these data show a

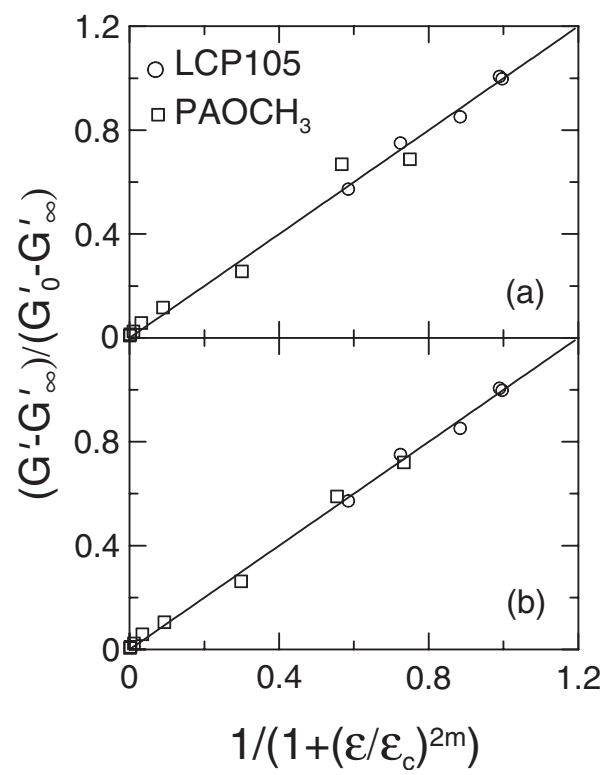

FIG. 13. Plot of the $G^{\prime}$ data for $\mathrm{LCP} 105$ and $\mathrm{PAOCH}_{3}$ as a function of reduced variables showing that the data for both compounds can be scaled on the same master curve. Panel (a) the $\mathrm{PAOCH}_{3}$ data are those of Fig. 1 of [42], taken at $1 \mathrm{rad} / \mathrm{s}$, and the LCP 105 data those of Fig. 11. Panel (b) the data for $\mathrm{PAOCH}_{3}$ correspond to the mean values deduced from the data reported as a function of frequency in Fig. 1(a) of [38] and the data for LCP 105 to those of Fig. 11. 
large dispersion which leads, especially for the weakest strains $(0.3 \%, 0.5 \%$, and $1 \%)$, to averaged values that are significantly different from the $G^{\prime}$ data taken at $1 \mathrm{rad} / \mathrm{s}$ only. Figure 13(b) shows the master curve obtained by using these averaged values. It can be seen that the quality of the master curve is appreciably higher than the one obtained with the previous data taken at $1 \mathrm{rad} / \mathrm{s}$.

Among the compounds we have studied, the Payne effect was so far observed only for the SCLCPs $\left(\mathrm{PAOCH}_{3}\right.$, LCP105). Thus, for the conventional polymers studied [PS $4000, \mathrm{PBuA}]$, the elastic plateau $G_{P L}$ decreases when the strain $\varepsilon$ increases, whereas the flow behavior observed for $G^{\prime \prime}$ remains unchanged $[22,23]$. This change in behavior reflects the slipping of the preglassy network on the silica slides, as explained in [23]. Note that the observation of the Payne effect for SCLCPs suggests that the preglassy network does not slip on the slides and that the anchoring of this network on the silica slides is probably stronger than for conventional polymers.

We close this section by noting that behaviors qualitatively similar to those of Figs. 10 and 12 have been observed in colloidal glasses and interpreted in terms of an extended Maxwell model in which the characteristic time is time dependent [51]. In this model, $G^{\prime \prime}$ passes through a maximum for $\varepsilon=\varepsilon_{c}$ and crosses $G^{\prime}$ at that point. However the data of $\mathrm{PAOCH}_{3}$ and LCP 105 show that $G^{\prime}$ is higher than $G^{\prime \prime}$ when $\varepsilon=\varepsilon_{c}$ and that $G^{\prime}$ and $G^{\prime \prime}$ cross at a value of $\varepsilon$ much higher than $\varepsilon_{c}$, indicating that the extended Maxwell model does not apply in these systems.

\section{Mechanical behavior of a polymer melt filled with silica particles}

The results obtained as a function of the pressure applied on the sample can be explained by a compression-assisted aggregation of the preglassy elastic clusters obtained at a given temperature by reducing the sample thickness. If this interpretation is correct, it should be possible to mimic these aggregation effects in a conventional PDMS melt filled with silica particles, the silica particles playing the role of the preglassy elastic clusters. We used silica particles from Degussa-Huls (A130) at a concentration $\mathrm{C}=6 \%(\mathrm{w} / \mathrm{w})$ and a PDMS sample from Gelest $\left(M_{\mathrm{w}}=17250 \mathrm{~g} / \mathrm{mol}\right)$. The material was prepared by mixing PDMS, chloroform, and the silica particles and by stirring the mixture for $\sim 12 \mathrm{~h}$. The chloroform allows to significantly reduce the viscosity of the mixture and thereby to favor, during the mixing, a more homogeneous dispersion of the particles. The chloroform was then slowly evaporated, and the resulting mixture degassed in order to eliminate any trace of chloroform and the air bubbles introduced in the mixture during the mixing. Figures 14 and 15 present the results obtained by filling the cell at room temperature, that is below the temperature $T_{0}$ corresponding to the gelation of the system when the temperature decreases. Figure 14 displays the behavior of $G^{\prime}$ and $G^{\prime \prime}$ as a function of frequency, indicating a gel-like response for the system, as for $\mathrm{PAOCH}_{3}$ (see Fig. 4). Figure 15 shows that the elastic plateau increases when a pressure is applied on the sample by bringing closer the sample-cover silica slide to the

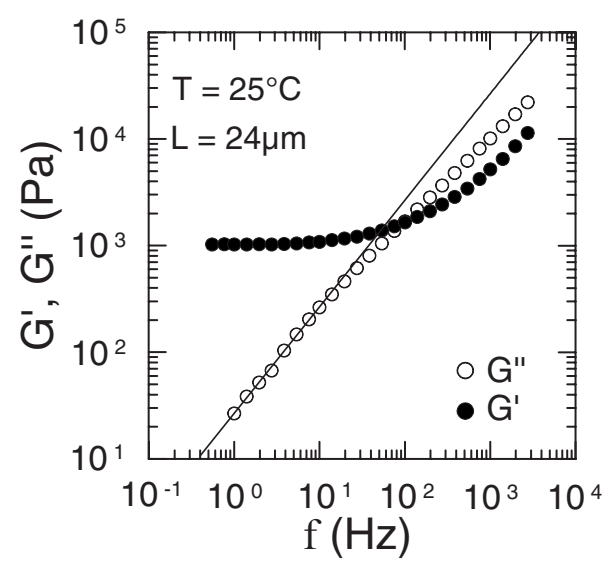

FIG. 14. Frequency dependence of the real $G^{\prime}$ and imaginary $G^{\prime \prime}$ parts of the shear modulus of the PDMS melt filled with the A130 silica particles at $T \sim 25{ }^{\circ} \mathrm{C}$. The sample thickness was $24 \mu \mathrm{m}$. The data show that the mechanical response of the system is gel-like $\left(G^{\prime} \sim\right.$ const and $\left.G^{\prime \prime} \sim f\right)$ in the low-frequency range. The straight line represents the behavior for $G^{\prime \prime}$ proportional to $f$.

sample-bearing silica slide, as for $\mathrm{PAOCH}_{3}$ (see Fig. 9). The variation in $G_{P L}$ as a function of the sample-thickness follows a power law, with an exponent $\sim 2$ that is close to the one obtained for $\mathrm{PAOCH}_{3}$.

So, it turns out that the experiments performed on the PDMS melt filled with silica particles reproduce the behavior previously observed in $\mathrm{PAOCH}_{3}$ and provide therefore an additional argument supporting our interpretation of the data for $\mathrm{PAOCH}_{3}$ and LCP105 in terms of preglassy elastic clusters.

\section{CONCLUSION}

In conclusion, we have studied the rheological response of $\mathrm{PAOCH}_{3}$ by examining the influence of the method used to fill the cell (compression method versus capillary method), of the filling temperature, and of the pressure strength used

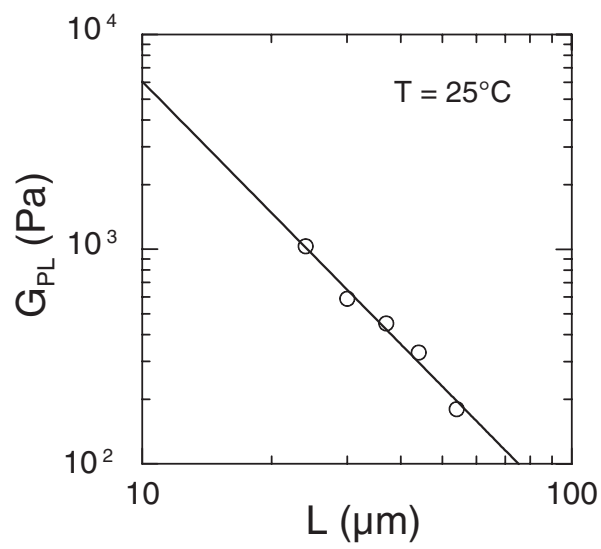

FIG. 15. Variation in the low-frequency elastic plateau of the PDMS melt filled with the A130 silica particles as a function of the thickness of the sample showing that a progressive reduction in the gap leads to an increase in $G_{P L}$. The straight line corresponds to the fit of the data with a power law. 
to fill the cell with the compression method. By filling the cell at high temperature, by gently bringing closer the sample-cover silica slide to the sample-bearing silica slide, we have observed that $\mathrm{PAOCH}_{3}$ exhibits a flow behavior in the temperature range located above a temperature $T_{0}$ and a gel-type behavior below $T_{0}$, for which the elastic response of $G^{\prime}$ is associated with the preglassy elastic clusters and the viscous response of $G^{\prime \prime}$ with the polymer chains. These results, which have also been obtained by filling the cell at high temperature with the capillary method, show that the compound is not a solidlike material with a type of unconventional elasticity characterized by $G^{\prime} \sim$ const and $G^{\prime \prime}$ $\sim$ const, which can be observed far away from any phase or glass transition, as claimed by Mendil et al. not only for $\mathrm{PAOCH}_{3}$ [39] but also for other materials [38]. The lowfrequency gel-type behavior we observed on $\mathrm{PAOCH}_{3}$ is similar to those we have already observed on various types of polymer melts including flexible polymer melts [22,23], showing that this behavior is independent of the chemical composition of the compounds. This gel-type behavior is that of a physical gel and is therefore transient and thermoreversible in contrast to the gel-like response of a solid or a chemical gel, which is not. It might be a general feature of polymer melts exhibiting a glass transition although further investigations on other polymer melts are needed to give a definite answer.

We have also shown that the strength of the pressure used to bring closer the sample-cover silica slide to the samplebearing silica slide plays a crucial role on the mechanical response. When the cell is filled at low temperature (i.e., when $T<T_{0}$ ), as in the experiments of Mendil et al., an increase in pressure applied on the sample transforms the gel-like response $\left(G^{\prime} \sim\right.$ const, $\left.G^{\prime \prime} \sim f\right)$ to a solidlike response $\left(G^{\prime} \sim\right.$ const, $G^{\prime \prime} \sim$ const $)$, similar to the one observed by Mendil et al. We have checked that this anomalous solidlike behavior does not change with time (over a period of at least three months) and persists when the temperature is increased, as in the experiment of Mendil et al., before disappearing gradually with time, when $T>T_{0}$, to recover the flow behavior. This latter is obtained after a time-period depending on the temperature difference $T-T_{0}$. The higher $T-T_{0}$, the faster the flow behavior is recovered. This anomalous solidlike behavior observed by filling the cell at low temperature does not reflect an intrinsic property of the melt but is rather the result of both the filling method and the filling temperature used. Consequently, we believe that the difference between the behavior of $\mathrm{PAOCH}_{3}$ observed by Mendil et al. $\left(G^{\prime}=\right.$ const, $G^{\prime \prime}=$ const $)$ and that of LCP105 $\left(G^{\prime}\right.$ =const, $G^{\prime \prime} \sim f$ ) stems from the difference in the pressure applied on the sample-cover silica slide to fill the cells and not from the chemical composition of the compounds as stressed by Mendil et al. in [41].

The behavior observed as a function of temperature after filling the cell at high temperature by gently bringing closer the sample-cover silica slide to the sample-bearing silica slide or by capillarity can be explained by the presence of preglassy elastic clusters, which start to "percolate" at the temperature $T_{0}$ mentioned above. $T_{0}$ plays a key role in the response of the sample: above $T_{0}$, the response is liquidlike, below $T_{0}$, the percolation of the elastic clusters gives rise to a low-frequency elastic response $\left(G^{\prime} \sim\right.$ const $)$, which is added to the flow response of the polymer melt, leading to an apparent gel-like behavior. $T_{0}$ depends on sample thickness and increases when the thickness decreases [22,23]. For a given thickness, $T_{0}$ should be an invariant since it represents the temperature at which the percolation of the elastic clusters occurs, but experimentally, $T_{0}$ is found to depend on the anchoring conditions. The anomalous elastic behavior $\left(G^{\prime}\right.$ =const, $G^{\prime \prime}=$ const), which occurs when one increases the pressure used to bring closer the sample-cover silica slide to the sample-bearing silica slide, is similar to that observed by Mendil et al. and can be explained by a compression-assisted aggregation of the preglassy elastic clusters present in the melt. We show that this compression-assisted aggregation can be mimicked by a conventional PDMS melt filled with silica particles, the silica particles playing the role of the preglassy elastic clusters. Additional evidence for the presence of the preglassy elastic clusters is given by the nonlinear response of the samples, which can be interpreted in terms of Payne effect.

We conclude this paper by underlying again that the geltype behavior $\left(G^{\prime}=\right.$ const, $\left.G^{\prime \prime} \sim f\right)$ observed here on SCLCPs $\left(\mathrm{PAOCH}_{3}\right.$ and LCP105) and previously on some conventional polymers (atactic polystyrene, polybutylacrylate) seems to be a generic effect of the glass transition. Therefore, this effect should also be observed in glass formers composed of small molecules if the size of the clusters is large enough to bridge the gap between the silica slides. As already stressed in [22], the presence of the preglassy clusters has a deep impact on the understanding of the glass transition because they invalidate the hypothesis of ergodicity above the glass transition.

\section{ACKNOWLEDGMENT}

We thank Elke Stibal-Fischer for the synthesis and the characterization of the $\mathrm{PAOCH}_{3}$ sample.
[1] E. Donth, The Glass Transition (Springer-Verlag, Berlin, 2001).

[2] M. D. Ediger, C. A. Angell, and S. R. Nagel, J. Phys. Chem. 100, 13200 (1996).

[3] H. Sillescu, J. Non-Cryst. Solids 243, 81 (1999).

[4] M. D. Ediger, Annu. Rev. Phys. Chem. 51, 99 (2000).
[5] E. W. Fischer, Physica A 201, 183 (1993).

[6] T. Kanaya, A. Patkowski, E. W. Fischer, J. Seils, H. Gläser, and K. Kaji, Acta Polym. 45, 137 (1994).

[7] T. Kanaya, A. Patkowski, E. W. Fischer, J. Seils, H. Gläser, and K. Kaji, Macromolecules 28, 7831 (1995).

[8] A. Patkowski, Th. Thurn-Albrecht, E. Banachowicz, W. Stef- 
fen, P. Bösecke, T. Narayanan, and E. W. Fischer, Phys. Rev. E 61, 6909 (2000).

[9] A. Patkowski, E. W. Fischer, W. Steffen, H. Gläser, M. Baumann, T. Ruths, and G. Meier, Phys. Rev. E 63, 061503 (2001).

[10] A. Patkowski, H. Gläser, T. Kanaya, and E. W. Fischer, Phys. Rev. E 64, 031503 (2001).

[11] E. W. Fischer, A. Bakai, A. Patkowski, W. Steffen, and L. Reinhardt, J. Non-Cryst. Solids 307-310, 584 (2002).

[12] R. Walkenhorst, J. C. Selser, and G. Piet, J. Chem. Phys. 109, 11043 (1998).

[13] M. T. Cicerone and M. D. Ediger, J. Chem. Phys. 104, 7210 (1996).

[14] L. A. Deschenes and D. A. Vanden Bout, J. Phys. Chem. B 106, 11438 (2002)

[15] A. Schob, F. Cichos, J. Schuster, and C. von Borczyskowski, Eur. Polym. J. 40, 1019 (2004).

[16] G. Diezemann, R. Böhmer, G. Hinze, and H. Sillescu, J. NonCryst. Solids 235-237, 121 (1998).

[17] U. Tracht, M. Wilhelm, A. Heuer, H. Feng, K. Schmidt-Rohr, and H. W. Spiess, Phys. Rev. Lett. 81, 2727 (1998).

[18] B. Schiener, R. V. Chamberlin, G. Diezemann, and R. Böhmer, J. Chem. Phys. 107, 7746 (1997).

[19] U. Schneider, P. Lunkenheimer, R. Brand, and A. Loidl, J. Non-Cryst. Solids 235-237, 173 (1998).

[20] J. L. Gallani, L. Hilliou, P. Martinoty, and P. Keller, Phys. Rev. Lett. 72, 2109 (1994).

[21] P. Martinoty, L. Hilliou, M. Mauzac, L. G. Benguigui, and D. Collin, Macromolecules 32, 1746 (1999).

[22] D. Collin and P. Martinoty, Physica A 320, 235 (2003).

[23] D. Collin and P. Martinoty, Eur. Phys. J. E 19, 87 (2006).

[24] J. L. Gallani, L. Hilliou, P. Martinoty, F. Doublet, and M. Mauzac, J. Phys. II 6, 443 (1996).

[25] J. Weilepp, P. Stein, N. Assfalg, H. Finkelmann, P. Martinoty, and H. R. Brand, Europhys. Lett. 47, 508 (1999).

[26] J. Weilepp, J. J. Zanna, N. Assfalg, P. Stein, L. Hilliou, M. Mauzac, H. Finkelmann, H. R. Brand, and P. Martinoty, Macromolecules 32, 4566 (1999).

[27] P. Stein, N. Assfalg, H. Finkelmann, and P. Martinoty, Eur. Phys. J. E 4, 255 (2001).

[28] J. J. Zanna, P. Stein, J. D. Marty, M. Mauzac, and P. Martinoty, Macromolecules 35, 5459 (2002).

[29] P. Martinoty, P. Stein, H. Finkelmann, H. Pleiner, and H. R.
Brand, Eur. Phys. J. E 14, 311 (2004).

[30] D. Rogez, H. Brandt, H. Finkelmann, and P. Martinoty, Macromol. Chem. Phys. 207, 735 (2006).

[31] D. Rogez, G. Francius, H. Finkelmann, and P. Martinoty, Eur. Phys. J. E 20, 369 (2006).

[32] D. Collin, G. Auernhammer, O. Gavat, P. Martinoty, and H. R. Brand, Macromol. Rapid Commun. 24, 737 (2003).

[33] G. K. Auernhammer, D. Collin, and P. Martinoty, J. Chem. Phys. 124, 204907 (2006).

[34] D. Collin, P. Lavalle, J. Mendez Garza, J. C. Voegel, P. Schaaf, and P. Martinoty, Macromolecules 37, 10195 (2004).

[35] H. R. Brand and K. Kawasaki, Physica A 324, 484 (2003).

[36] L. Noirez, Phys. Rev. E 72, 051701 (2005).

[37] H. Mendil, P. Baroni, and L. Noirez, Europhys. Lett. 72, 983 (2005).

[38] H. Mendil, P. Baroni, and L. Noirez, Eur. Phys. J. E 19, 77 (2006).

[39] H. Mendil, L. Noirez, P. Baroni, and I. Grillo, Phys. Rev. Lett. 96, 077801 (2006).

[40] D. Collin and P. Martinoty, Phys. Rev. Lett. 98, 249801(C) (2007).

[41] H. Mendil, L. Noirez, P. Baroni, and I. Grillo, Phys. Rev. Lett. 98, 249802(R) (2007).

[42] H. Mendil, P. Baroni, and L. Noirez, Eur. Phys. J. E 19, 99 (2006).

[43] See, for example, J. D. Ferry, Viscoelastic Properties of Polymers, 3rd ed. (Wiley, New York, 1980); G. Strobl, The Physics of Polymers (Springer, Berlin, 1996).

[44] P. G. de Gennes, C. R. Phys. 3, 1263 (2002).

[45] See, for example, M. E. Helgeson, N. J. Wagner, and D. Vlassopoulos, J. Rheol. 51, 297 (2007).

[46] See, for example, H. Soenen, H. Berghmans, H. H. Winter, and N. Overbergh, Polymer 38, 5653 (1997).

[47] Figures 13 and 14 of [23] are not in contradiction with Fig. 1 of [40], in contrast to the claim of Mendil et al. in [41].

[48] A. R. Payne, J. Appl. Polym. Sci. 6, 368 (1962).

[49] G. Kraus, J. Appl. Polym. Sci.: Appl. Polym. Symp. 39, 75 (1984).

[50] G. Huber and T. A. Vilgis, Kautsch. Gummi Kunstst. 52, 102 (1999).

[51] See, for example, K. Miyazaki, H. M. Wyss, D. A. Weitz, and D. R. Reichman, Europhys. Lett. 75, 915 (2006). 